\title{
HLA-B*58:01 allele is strongly associated with allopurinol-induced severe cutaneous adverse reactions in a Thai population
}

\author{
Thawinee Jantararoungtong ${ }^{1 *}$, Ticha Rerkpattanapipat ${ }^{2}$, Santirat Prommas ${ }^{1}$, Napatrupron Koomdee ${ }^{1}$, \\ Siwalee Santon ${ }^{1}$, Montri Chammanphol ${ }^{1}$, Apichaya Puangpetch ${ }^{1}$, Chonlaphat Sukasem ${ }^{1}$
}

From 6th Drug Hypersensitivity Meeting (DHM 6)

Bern, Switzerland. 9-12 April 2014

\section{Background}

Allopurinol has been reported as the most frequent causes of SCARs (severe cutaneous adverse reactions) in Thailand. Recent publications have shown that HLAB*58:01 allele is a strong marker for allopurinol-induced SJS/TEN (Stevens-Johnson syndrome/toxic epidermal necrolysis). The aim of this study was to clarify the association of allopurinol-induced SCARs with the HLA-B*58:01 allele in Thai patients.

\section{Method}

To investigate this relationship, we performed PCR-SSOP (sequence specific oligonucleotide probe) on allopurinoltolerant controls $(\mathrm{n}=56)$ and patients affected by allopurinol-induced SCARs $(n=14)$. Among of allopurinol-induced SCARs, including 3 patients with allopurinol-induced DRESS (drug reaction with eosinophilia and systemic symptoms), 9 patients with SJS/TEN and 2 patients with MPE (maculo-papular exanthema) were included. The presence of HLA-B*58:01 allele were genotyped by PCRSSOP method at Laboratory for Pharmacogenomics, Ramathibodi Hospital.

\section{Results}

Of the 14 patients with allopurinol-induced SCARs, 13 (92.8\%) patients (3 DRESS, 9 SJS/TEN and one severe MPE) had HLA-B*58:01 while only $6(10.71 \%)$ of the allopurinol-tolerant controls had this allele. The risk of allopurinol-induced SCARs was significantly higher in the patients with HLA-B*58:01 with an OR (odd ratios) of 108.33 (95\% CI 11.96-980.82, $\mathrm{p}<10-6$ ). When compared with normal population, HLA-B*58:01 was associated with a higher risk of SCARs, both DRESS (OR: 80, 95\% CI 3.42-372.87) and SJS/TEN (OR: 217.26, 95\%CI 12.41-925.35).

\section{Conclusion}

In this study we confirmed that HLA-B*58:01 allele is strongly associated with allopurinol-induced SCARs in Thai population. Therefore, screening tests for HLAB*58:01 allele in patients who will be treated with allopurinol will be clinically helpful in preventing the risk of developing SCARs.

\section{Authors' details \\ ${ }^{1}$ Ramathibodi Hospital, Laboratory for Pharmacogenomics, Thailand. \\ ${ }^{2}$ Ramathibodi Hospital, Medicine Department, Thailand.}

Published: 18 July 2014

\section{doi:10.1186/2045-7022-4-S3-P120}

Cite this article as: Jantararoungtong et al:: HLA-B*58:01 allele is strongly associated with allopurinol-induced severe cutaneous adverse reactions in a Thai population. Clinical and Translational Allergy 2014 4(Suppl 3): P120.

${ }^{1}$ Ramathibodi Hospital, Laboratory for Pharmacogenomics, Thailand

Full list of author information is available at the end of the article 